FOUNDATIONS

ADVANCES

ISSN 2053-2733

Received 21 January 2021

Accepted 21 January 2021

Keywords: electron density; data quality; charge density; powder diffraction; MYTHEN detector.

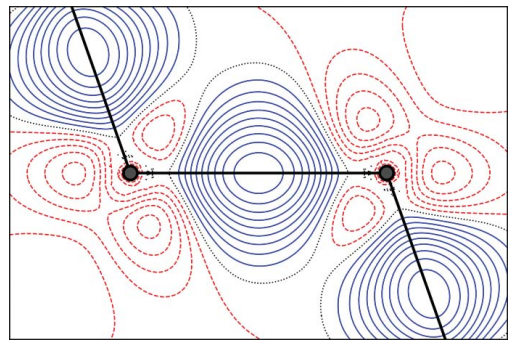

\section{Nothing trumps good data}

\author{
A. Alan Pinkerton*
}

Department of Chemistry and Biochemistry, University of Toledo, Toledo, OH 43606, USA. *Correspondence e-mail: a.pinkerton@utoledo.edu

$\mathrm{X}$-rays are scattered by electrons, and the Fourier transform of the X-ray diffraction pattern obtained from a periodic (read crystalline) material is the electron-density distribution in that solid. The details of that distribution depend on the quality of the diffraction data, but even in 1915 it was realized that subatomic details should be available from data of sufficient quality (Debye, 1915). However, it would take decades of hardware and software development to make that a reality.

Although much of the driving force in hardware development could be considered to be reduction in the time required for a diffraction experiment, a frequent consequence was an improvement in data quality. Thus, as the intensity of X-ray beams increased, the ability to detect weaker reflections became possible. As detection of the scattered rays moved from photographic film to point detectors to area detectors, the number of reflections measurable in any one experiment increased dramatically. The impact on the science can be seen from the concomitant increase in sophistication of the structural models - from spherical stationary atoms to isotropic then anisotropic and currently (Birkedal et al., 2004) anharmonic atomic displacement parameters, and then to aspherical atoms. For light-atom systems, the current state of the art uses a multipolar expansion centered at the atomic nucleus to describe the valence electron distribution (Hansen \& Coppens, 1978), and allows polarization of the core electron density to also be observed (Bindzus et al., 2014). For heavy-atom systems, several sets of multipolar expansions with different radial functions are now used to model the different valence shells and the outer core region (Gianopoulos et al., 2019). Of course, the introduction of new technology does not automatically provide better data; however, the crystallographic community has always been very conscientious in the evaluation and benchmarking of new hardware and software.

The majority of charge-density studies have been carried out using diffraction data from single crystals. However, when studying inorganic crystals with small unit cells, there are two serious problems that may arise. The first of these, extinction, typically leads to serious underestimation of strong reflections. The second problem is twinning, leading to superposition of diffraction patterns. Today, neither of these is considered a major problem for routine structure determination, but they still present serious challenges for charge-density studies. However, if such crystals are ground to a fine powder, then the effect of extinction is largely eliminated from the diffraction pattern, and reflections from all twin domains perfectly overlap. But now the challenge is to extract the $3 \mathrm{D}$ charge density from a $1 \mathrm{D}$ diffraction pattern. In recent years, significant progress has been made in this area. Measurements on diamond powder provided a proof of principle for the use of powder diffraction data to obtain the charge-density distribution in close agreement with theory (including core polarization), as well as benchmarking a new vacuum imageplate detector (Bindzus et al., 2014).

In this issue, Svane et al. (2021) continue the important tradition of evaluating and benchmarking new equipment and techniques, in particular with regard to the use of powder diffraction data to determine the experimental charge density. The microstrip detector (MYTHEN) has a sharper point-spread function than and potentially a similar dynamic range as an image plate (Bergamaschi et al., 2010). However, this dynamic range is significantly reduced by non-uniformity of the X-ray response. Recently, the dynamic range has been largely restored by a statistical approach to the response correction (Kato \& Shigeta, 2020). Svane et al. (2021) have used the reported image-plate results for diamond as a benchmark to evaluate the data from the MYTHEN detector with the new correction. They used the same Hansen-Coppens/Rietveld strategy as before, and the 


\section{scientific commentaries}

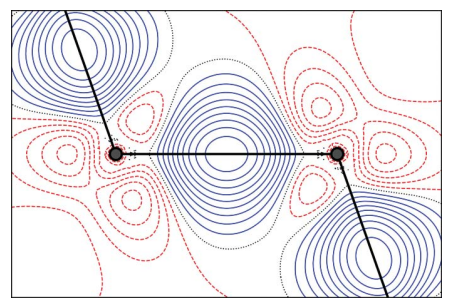

Figure 1

Static deformation density in the (110) plane of diamond with contours at 0.05 e $\AA^{-3}$ (reproduced from Svane et al., 2021).

final refinement model agreed very well with that reported from the image-plate data with comparable very low residual densities (Fig. 1). Hence, this faster and easier to use detector is an excellent replacement for the image plate used previously (Svane et al., 2021).

As mentioned above, the point-spread function of MYTHEN is sharper than that of an image plate. This suggests that studies on systems with lower symmetry and larger unit cells, or indeed molecular systems, might well be tractable owing to a reduction in reflection overlap. Thus Svane et al. have carried out studies to compare MYTHEN and imageplate powder data with single-crystal results on urea (Birkedal et al., 2004) and xylitol (Madsen et al., 2004). Although not as convincing as the diamond results, the outcome is very encouraging. Not surprisingly, the most difficulty was encountered with the extraction of structure factors at high angles owing to the population density of reflections and the concomitant problem of reflection overlap.

Although the use of powder data might not be the method of choice for charge-density studies on most molecular systems, Svane et al. have shown that it is clearly a very powerful tool for the study of small inorganic systems where extinction is a well known issue for single-crystal specimens.

\section{References}

Bergamaschi, A., Cervellino, A., Dinapoli, R., Gozzo, F., Henrich, B., Johnson, I., Kraft, P., Mozzanica, A., Schmitt, B. \& Shi, X. (2010). J. Synchrotron Rad. 17, 653-668.

Bindzus, N., Straas $\varnothing$, T., Wahlberg, N., Becker, J., Bjerg, L., Lock, N., Dippel, A.-C. \& Iversen, B. B. (2014). Acta Cryst. A70, 39-48.

Birkedal, H., Madsen, D., Mathiesen, R. H., Knudsen, K., Weber, H.-P., Pattison, P. \& Schwarzenbach, D. (2004). Acta Cryst. A60, 371-381.

Debye, P. (1915). Ann. Phys. 351, 809-823.

Gianopoulos, C. G., Zhurov, V. V. \& Pinkerton, A. A. (2019). IUCrJ, 6, 895-908.

Hansen, N. K. \& Coppens, P. (1978). Acta Cryst. A34, 909-921.

Kato, K. \& Shigeta, K. (2020). J. Synchrotron Rad. 27, 1172-1179.

Madsen, A. Ø., Sørensen, H. O., Flensburg, C., Stewart, R. F. \& Larsen, S. (2004). Acta Cryst. A60, 550-561.

Svane, B., Tolborg, K., Kato, K. \& Iversen, B. B. (2021). Acta Cryst. A77, 85-95. 